\title{
Representing future generations in public participation procedures regarding the siting of a nuclear waste repository
}

\author{
Lisa P. Hamacher \\ Bucerius Law School, Hamburg, 20355, Germany \\ Correspondence: Lisa P. Hamacher (lisa.hamacher@law-school.de) \\ Published: 10 November 2021
}

\begin{abstract}
State decisions regarding a repository for high-level radioactive waste have an extraordinary intergenerational significance. The academic legal discussion has increasingly strengthened the status of future generations in constitutional law. In its recent decision on the German Climate Protection Act, the Federal Constitutional Court equally emphasised that state actors have an obligation to protect future generations. Fundamental rights of future generations thus have an anticipated effect in the present. In general, the legislator is free to choose the appropriate means to protect these rights. The interests of future generations may be promoted by substantive or procedural law. The German Site Selection Act (StandAG), however, makes use of procedural protections to a significant extent. Including the interests of future generations in the existing public participation procedures and participation bodies is, therefore, crucial for the effective protection of future generations.

The presentation examines to which extent the current legal framework for the site selection for a high-level radioactive waste repository in Germany enables an effective representation of the future generations' interests. Existing publications (Appel, 2005; Rose, 2018; Kloepfer, 1993) name various characteristics of effective "intergenerational institutions". Accordingly, these institutions should be independent, exist long-term, serve future interests solely or primarily, and have strong rights vis-à-vis decision-makers; however, German constitutional law, namely the principle of democracy, limits the design of such institutions. Not all of the abovementioned characteristics can be combined. Nevertheless, the constitution enables means to include and promote the interests of future generations in decision-making procedures, which are not fully exploited by the German Site Selection Act. The participation procedure includes several groups which could promote intergenerationally just decisions, namely environmental associations, the public and representatives of the "young generation"; however, none of these stakeholders are "intergenerational institutions" in the abovementioned sense. Subsequently, the presentation proposes various reforms: improving the sustainability impact assessment during the legislative procedure, the implementation of an "intergenerational impact assessment" and an ombudsperson for future concerns, who could be affiliated with the National Citizens' Oversight Committee (Nationales Begleitgremium).
\end{abstract}

Kurzfassung. Staatliche Entscheidungen zur Endlagerung hochradioaktiver Abfälle haben eine außerordentliche generationenübergreifende Bedeutung. Die rechtswissenschaftliche Diskussion hat den Status künftiger Generationen im Verfassungsrecht zunehmend gestärkt. So hat auch das Bundesverfassungsgericht in seiner kürzlich ergangenen Entscheidung zum Klimaschutzgesetz betont, dass staatliche Akteure zum Schutz künftiger Generationen verpflichtet sind. Grundrechte künftiger Generationen entfalten damit eine Vorwirkung in der Gegenwart. Grundsätzlich ist der Gesetzgeber in der Wahl der zum Schutz dieser Rechte geeigneten Mittel frei. Die Interessen künftiger Generationen können durch materielles oder Verfahrens- und Organisationsrecht wahrgenommen werden. Das Standortauswahlgesetz (StandAG) bedient sich jedoch in erheblichem Umfang des Grundrechtsschutzes durch Organisation und Verfahren. Die Einbeziehung der Interessen künftiger Generationen in die bestehenden Verfahren der Öffentlichkeitsbeteiligung und der Beteiligungsgremien ist daher für einen wirksamen Schutz künftiger Generationen von entscheidender Bedeutung. 
Im Vortrag wird untersucht, inwieweit der derzeitige rechtliche Rahmen für die Standortauswahl eines Endlagers für hochradioaktive Abfälle in Deutschland eine effektive Einbeziehung der Belange künftiger Generationen ermöglicht. In der Literatur (Appel, 2005; Rose, 2018; Kloepfer, 1993) werden verschiedene Merkmale effektiver „Zukunftsinstitutionen“ genannt. Demnach sollten diese Institutionen unabhängig sein, langfristig bestehen, ausschließlich oder vorrangig künftigen Interessen dienen und mit starken Rechten gegenüber Entscheidungsträgern ausgestattet sein; allerdings setzt das deutsche Verfassungsrecht, namentlich das Demokratieprinzip, der Ausgestaltung solcher Institutionen Grenzen. Nicht alle der aufgeführten Merkmale lassen sich miteinander kombinieren. Dennoch bietet das Grundgesetz Möglichkeiten, die Interessen künftiger Generationen in Entscheidungsverfahren einzubeziehen und zu fördern. Diese schöpft das Standortauswahlgesetz nicht voll aus. Das Beteiligungsverfahren bezieht zwar etliche Gruppen ein, die generationenübergreifend für gerechte Entscheidungen eintreten könnten, nämlich Umweltverbände, die Öffentlichkeit und Vertreterinnen und Vertreter der jungen Generationen; allerdings ist keiner dieser Akteure eine „Zukunftsinstitution“ im oben angeführten Sinn. Abschließend werden im Vortrag verschiedene Reformen vorgestellt: die Verbesserung der Nachhaltigkeitsprüfung im Gesetzgebungsverfahren, die Einführung einer „Nachweltverträglichkeitsprüfung “ und eine Ombudsperson für Zukunftsfragen, die dem Nationalen Begleitgremium zugeordnet sein könnte.

\section{References}

Appel, I.: Staatliche Zukunfts- und Entwicklungsvorsorge, Mohr Siebeck, Tübingen, Germany, 2005.

Kloepfer, M.: Langzeitverantwortung im Umweltstaat, in: Langzeitverantwortung im Umweltstaat, edited by: Gethmann, C. F. and Nutzinger, H. G., Economic, Bonn, Germany, 22-41, 1993.
Rose, M.: Zukünftige Generationen in der heutigen Demokratie: Theorie und Praxis der Proxy-Repräsentation, Springer VS, Wiesbaden, Germany, https://doi.org/10.1007/978-3-658-188467, 2018. 